Results: We detected 1440 differentially expressed genes between dcSSc vs $\mathrm{HC}$ and 225 between IcSSc and $\mathrm{HC}$ respectively $(\mathrm{p} \leq 0.01$; log2 ratio $\geq 0.5$, figure 1). Among those, in dcSSc 1076 were upregulated (e.g. MMP9, IL1R2, FLT3, MIF, TLR9) and 364 were downregulated (e.g. TGFBR1, CD44, CD244, HLADRA, HLA-G). In IcSSc 160 transcripts were upregulated (e.g. CCL2, WNT5B, MMP17) and 65 were downregulated (e.g. KLF11, IRAK2). We identified 123 commonly deregulated genes between SSc subgroups (e.g. CCL3, CD14, IL27, MMP17). Principal component analysis showed close clustering within SSc subgroups and clear separation from healthy controls. Pathway analysis revealed alterations in several biological processes important in fibrogenesis including antigen presentation, MIF-induced immune responses, TGF- $\beta$, NOTCH and WNT signalling pathways. qPCR analysis further confirmed differences in gene expression on mRNA level $(\mathrm{n} \mathrm{HC}=8, \mathrm{n} \mathrm{SSc}=25, \mathrm{p} \leq 0.05)$.
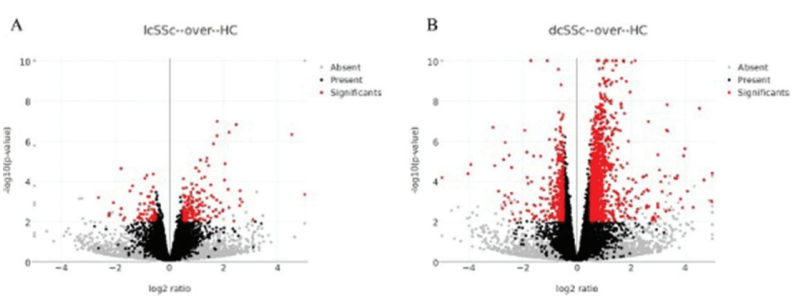

Abstract FRI0420 - Figure 1. Volcano plots representing differently expressed genes in IcSSc vs $\mathrm{HC}(\mathrm{A})$ and dcSSc vs $\mathrm{HC}(\mathrm{B})$. Red dots stand for significantly deregulated transcripts $(p \leq 0.01 ; \log 2$ ratio $\geq 0.5)$

Conclusions: To our knowledge, this is the first global transcriptome analysis of peripheral blood $\mathrm{CD} 14^{+}$monocytes in SSc. Our results suggest an initial activation of monocytes in peripheral blood, which might be further translated into novel cellular biomarker of the disease and potentially used for distinguishing between responders and non-responders to a novel treatment in future clinical trials.

Disclosure of Interest: M. Rudnik: None declared, M. Stellato: None declared, P. Blyszczuk: None declared, O. Distler Grant/research support from: Actelion, Bayer, Boehringer Ingelheim, Mitsubishi Tanabe Pharma and Roche, Consultant for: Actelion, Bayer, Biogenldec, Boehringer Ingelheim, ChemomAb, espeRare foundation, Genentech/Roche, GSK, Inventiva, Italfarmaco, Lilly, medac, Medlmmune, Mitsubishi Tanabe Pharma, Pharmacyclics, Novartis, Pfizer, Sanofi, Sinoxa and UCB, G. Kania Grant/research support from: Bayer Pharma AG, Actelion

DOI: 10.1136/annrheumdis-2018-eular.4504

\section{FRI0421 TOLL LIKE RECEPTOR-7/8 ACTIVATION EXACERBATES MURINE EXPERIMENTAL AUTOIMMUNE MYOSITIS}

R. De Lorenzo ${ }^{1}$, C. Sciorati ${ }^{1}$, A. Monno ${ }^{1}$, M.G. Doglio ${ }^{1}$, E. Rigamonti ${ }^{1}$,

D. Ascherman ${ }^{2}$, A. Manfredi ${ }^{1}$, P. Rovere-Querini ${ }^{1}{ }^{1}$ San Raffaele Scientific Institute, Milan, Italy, ${ }^{2}$ University of Miami, Miami, USA

Background: Type I interferon (IFN)-regulated proteins are upregulated in muscle and skin tissues of patients with idiopathic inflammatory myopathies (IIM). ${ }^{1}$ Type I IFN induction might rely upon the activation of toll-like receptors (TLRs). ${ }^{2}$ Specifically, TLR-7/8 is indeed upregulated in infiltrating leukocytes and muscle tissue of IIM patients. ${ }^{3}$

Objectives: To investigate whether the activation of TLR-7/8 and type I IFN influences the natural history of the IIM.

Methods: Experimental autoimmune myositis (EAM) was induced by injection of the amino-terminal portion of the murine Histydil t-RNA synthetase (HisRS). Disease activity was compared in the presence or absence of the TLR-7/8 agonist R848 in wild-type mice and in mice that failed to express the IFNab receptor (IFNabR null).

Results: EAM induced by a single intramuscular immunisation with HisRS spontaneously abated after 7-8 weeks. In contrast, the levels of anti-HisRS autoantibodies, endomysial/perimysial leukocyte infiltration and myofiber regeneration persisted until the end of the follow-up period (22 weeks after immunisation) in mice immunised with HisRS in the presence of R848. Myofiber MHC class I molecules were detectable in HisRS +R848 immunised mice only. Muscle MHC expression occurred in parallel with leukocyte infiltration. Type I IFN was necessary for the prolonged autoantibody response to occur and for the spreading of the autoimmune response, as demonstrated using IFNabR null mice.

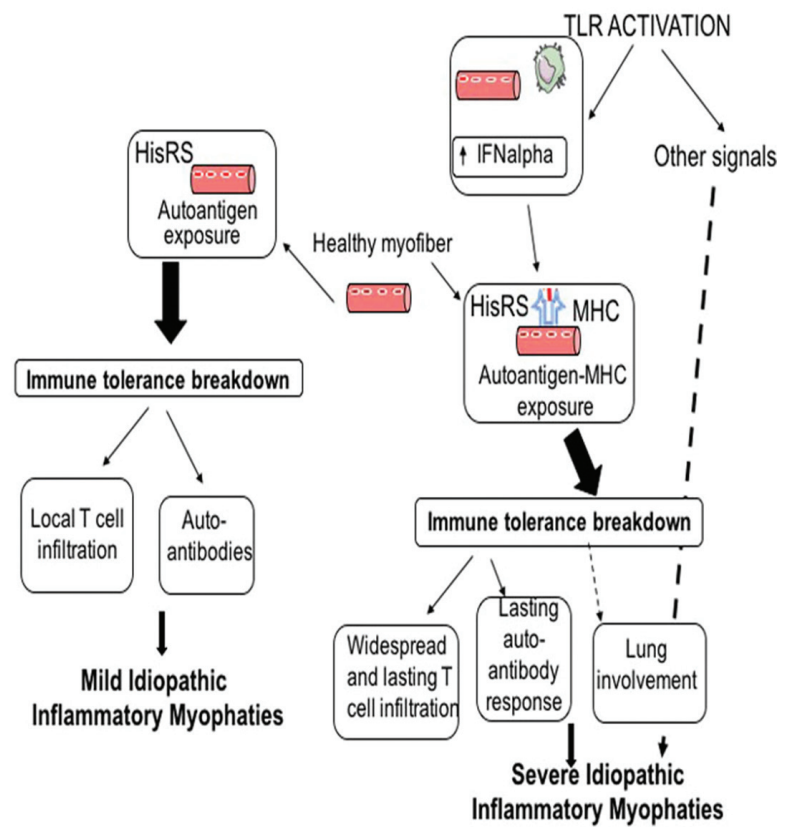

Abstract FRI0421 - Figure 1. Role of TLR7/8 activation in HisRS-induced myositis

Conclusions: TLR7/8 activation is needed to induce and maintain a systemic autoimmune response against the skeletal muscle. This EAM model reproduces many characteristics of human IIM and may represent a tool for pre-clinical studies.

\section{REFERENCES:}

[1] Wenzel J, Scheler M, Bieber T, Tuting T. Evidence for a role of type I interferons in the pathogenesis of dermatomyositis. $\mathrm{Br} \mathrm{J}$ Dermatol. 2005;153:462-463. [author reply 463-464]

[2] Drexlerand SK, Foxwell BM. The role of toll-like receptors in chronic inflammation. Int J Biochem Cell Biol. 2010;42:506-518.

[3] A. Tournadre, V. Lenief, A. Eljaafari, and P. Miossec. Immature muscle precursors are a source of interferon-beta in myositis: role of Toll-like receptor 3 activation and contribution to HLA class I up-regulation. Arthritis and rheumatism. 64:533-541 (2012).

Disclosure of Interest: None declared

DOI: 10.1136/annrheumdis-2018-eular.6432

\section{FRI0422 ROLE OF THE PROLYL 3-HYDROXYLASE LEPREL1 IN FIBROSIS}

H. Lopez ${ }^{1,2}$, B. Abdi Ahmed ${ }^{1}$, D. Abraham ${ }^{1}$, L. Bozec ${ }^{3}$, C. Denton ${ }^{1}$, A. Strange ${ }^{3}$, G. Martin ${ }^{4}$, R. Stratton ${ }^{1} .{ }^{1}$ Centre for Rheumatology and Connective Tissue Diseases, UCL, London, UK; ${ }^{2}$ Murigenics, Valejo CA, USA; ${ }^{3}$ Eastman Centre for Postgraduate Research, UCL, London, UK; ${ }^{4}$ Riptide Bioscience, San Francisco CA, USA

Background: Three prolyl 3-hydroxylase enzymes, LEPRE1, LEPREL1 and LEPREL2, are known to modify prolines in certain sequences in the C-terminal helical region of the polypeptide chains of procollagens converting them to 3 hydroxyproline residues. This modification appears to facilitate correct alignment of the chains in forming the triple helical domains of the procollagen molecules prior to secretion. Increased deposition of triple helical collagen and other extracellular matrix (ECM) proteins by activated fibroblasts underlies pathological fibrosis in systemic sclerosis (SSc), and may be dependent on prolyl 3-hydroxylase activity as a rate-limiting step.

Objectives: The objectives of this study were: 1 ) to screen candidate genes with large first introns containing regulatory elements for association with systemic sclerosis (SSc) through copy number variation (CNV), 2) to study the lead candidate gene LEPREL1 further, as a fibrosis-related factor in genetically modfied mice subject to bleomycin induced skin fibrosis, 3 ) to determine the levels of 